\title{
Intrinsic Halide Segregation at Nanometer Scale Determines the High Efficiency of Mixed Cation/Mixed Halide Perovskite Solar Cells
}

\author{
Paul Gratia, ${ }^{\dagger}$ Giulia Grancini, ${ }^{*}{ }^{\dagger}$ Jean-Nicolas Audinot, ${ }^{\dagger}$ Je Xavier Jeanbourquin, ${ }^{\S}$ Edoardo Mosconi, ${ }^{\|}$
}

Iwan Zimmermann, ${ }^{\dagger}$ David Dowsett, ${ }^{\ddagger}$ Yonghui Lee, ${ }^{\dagger}$ Michael Grätzel, ${ }^{\perp}$ Filippo De Angelis, ${ }^{\prime, \#}$

Kevin Sivula, ${ }^{\S}$ Tom Wirtz, ${ }^{\ddagger}$ and Mohammad Khaja Nazeeruddin ${ }^{*}{ }^{\dagger}$

${ }^{\dagger}$ Group for Molecular Engineering of Functional Materials (GMF), Institute of Chemical Sciences and Engineering, Swiss Federal Institute of Technology, CH-1951 Sion, Switzerland

${ }^{\ddagger}$ Advanced Instrumentation for Ion Nano-Analytics (AINA), Materials Research and Technology Department, Luxembourg Institute of Science and Technology (LIST), L-4422 Belvaux, Luxembourg

${ }^{\S}$ Molecular Engineering of Optoelectronic Nanomaterials Lab (LIMNO), Swiss Federal Institute of Technology, CH 1015 Lausanne, Switzerland

"Computational Laboratory for Hybrid/Organic Photovoltaics (CLHYO), CNR-ISTM, Via Elce di Sotto 8, I-06123 Perugia, Italy

${ }^{\perp}$ Laboratory for Photonics and Interfaces (LPI), Institute of Chemical Sciences and Engineering, Swiss Federal Institute of

Technology, CH-1015 Lausanne, Switzerland

${ }^{\#}$ Computet, Istituto Italiano di Tecnologia, Via Morego 30, I-16163 Genova, Italy

Supporting Information

ABSTRACT: Compositional engineering of a mixed cation/mixed halide perovskite in the form of (FAP$\left.\mathrm{bI}_{3}\right)_{0.85}\left(\mathrm{MAPbBr}_{3}\right)_{0.15}$ is one of the most effective strategies to obtain record-efficiency perovskite solar cells. However, the perovskite self-organization upon crystallization and the final elemental distribution, which are paramount for device optimization, are still poorly understood. Here we map the nanoscale charge carrier and elemental distribution of mixed perovskite films yielding $20 \%$ efficient devices. Combining a novel in-house-developed highresolution helium ion microscope coupled with a secondary ion mass spectrometer (HIM-SIMS) with Kelvin probe force microscopy (KPFM), we demonstrate that part of the mixed perovskite film intrinsically segregates into iodide-rich perovskite nanodomains on a length scale of up to a few hundred nanometers. Thus, the homogeneity of the film is disrupted, leading to a variation in the optical properties at the micrometer scale. Our results provide unprecedented understanding of the nanoscale perovskite composition.

P erovskite photovoltaics (PVs), which appear in the list of the "Top 10 Emerging Technologies of 2016", 1 promise to be a major player in the near-future carbon-free energy landscape. Among the large variety of perovskite compositions, the mixed cation/mixed halide $\left(\mathrm{FAPbI}_{3}\right)_{0.85}\left(\mathrm{MAPbBr}_{3}\right)_{0.15}$ perovskites $(\mathrm{MA}=$ methylammonium; $\mathrm{FA}=$ formamidinium $)$ currently hold the lead since they have repeatedly proven to yield high power conversion efficiencies (PCEs) beyond 20\%, ${ }^{2,3}$ competing with established thin-film PV technologies. This has been achieved with exceptional ease by tuning the material's optoelectronic properties and processing upon simple chemical substitution. ${ }^{4-6}$ Efforts to optimize pure $\mathrm{MAPbI}_{3}{ }^{7}$ by composi- tional engineering of cations (e.g., substitution of MA by FA) and anions (e.g., introducing a small amount of $\mathrm{Br}$ ) along with the addition of excess lead iodide have indeed induced a breakthrough in device efficiency and reproducibility. ${ }^{8,5}$ Recent developments even include triple cation structures containing cesium, MA, and FA. ${ }^{9}$ The higher efficiency compared with single $\mathrm{MAPbI}_{3}$ perovskite structures has been generically attributed to the improved crystal quality of the film, ${ }^{3}$ but the exact reasons remain under intense debate. Previous reports have found that light-induced halide segregation in $\mathrm{MAPbBr}_{x} \mathrm{I}_{3-x}$ (no FA cation), ${ }^{10,11}$ iodine migration in $\mathrm{MAPbI}_{3}$ perovskites, ${ }^{12}$ and electric-field-induced halide migration $^{13}$ have enormous impacts on the device behavior. Nevertheless, a rational investigation of the intrinsic mixed cation/mixed halide $\left(\mathrm{FAPbI}_{3}\right)_{0.85}\left(\mathrm{MAPbBr}_{3}\right)_{0.15}$ perovskite composition is still missing. In this work, we provide in-depth insight into the local elemental composition and structuraloptical properties of high-efficiency mixed perovskite devices by using advanced nanoscale mapping techniques.

A novel in-house-developed helium ion microscope coupled to a secondary ion mass spectrometer (HIM-SIMS) revealed that the mixed perovskite film self-organizes to form large domains of pure iodide perovskite (up to a few hundred nanometers in size) spatially segregated from the mixed iodide/ bromide phases. Such nanoscale segregation is responsible for driving local charge distribution and charge accumulation in the iodide rich domains, as revealed by Kelvin probe force microscopy (KPFM) mapping.

High-efficiency $\left(\mathrm{FAPbI}_{3}\right)_{0.85}\left(\mathrm{MAPbBr}_{3}\right)_{0.15}$ solar cells were fabricated using a previously described protocol. ${ }^{3}$ Device current-voltage $(I-V)$ characteristics showing a champion device efficiency of $20.4 \%$ and device statistics are plotted in

Received: September 24, 2016

Published: November 29, 2016 

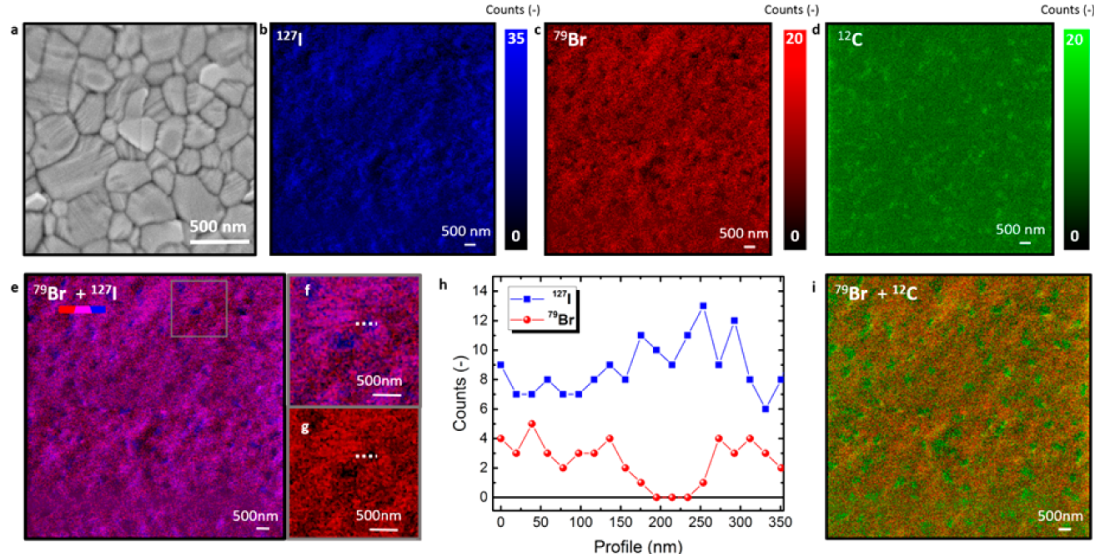

Figure 1. Elemental nanoscale HIM-SIMS mapping. (a) SEM surface image of $\left(\mathrm{FAPbI}_{3}\right)_{0.85}\left(\mathrm{MAPbBr}_{3}\right)_{0.15}$ perovskite deposited on a mesoporous $\mathrm{TiO}_{2}$ scaffold. (b-d) HIM-SIMS elemental mapping of ${ }^{127} \mathrm{I}$ (blue), ${ }^{79} \mathrm{Br}$ (red), and ${ }^{12} \mathrm{C}$ (green) across a $10 \mu \mathrm{m} \times 10 \mu \mathrm{m}$ area. (e) Overlap of the ${ }^{79} \mathrm{Br}$ (red) and ${ }^{127} \mathrm{I}$ (blue) signals. The "blue" spots have low ${ }^{79} \mathrm{Br}$ and high ${ }^{127} \mathrm{I}$ intensities. (f, g) Zoomed views of (f) combined ${ }^{79} \mathrm{Br}$ and ${ }^{127} \mathrm{I}$ and $(\mathrm{g}){ }^{79} \mathrm{Br}$ alone. (h) Profiles of the ${ }^{79} \mathrm{Br}$ and ${ }^{127} \mathrm{I}$ signals across the dashed lines in $(\mathrm{f})$ and $(\mathrm{g})$. (i) Overlap of ${ }^{79} \mathrm{Br}$ and ${ }^{12} \mathrm{C}$ signals revealing that the carbon hotspots in (d) correspond to ${ }^{79} \mathrm{Br}$ signal minima.
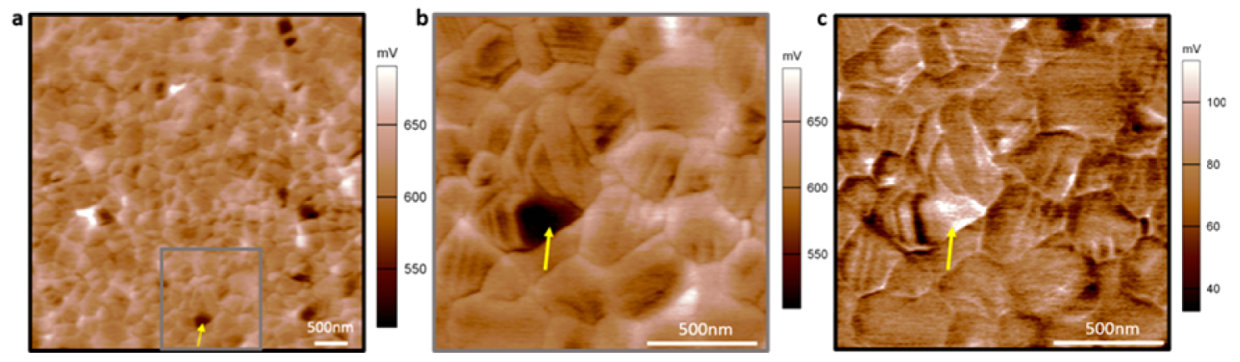

Figure 2. KPFM measurements in the dark and under illumination. (a) A $5 \mu \mathrm{m} \times 5 \mu \mathrm{m}$ surface potential map of the capping layer of a mixed perovskite solar cell (no cathode) in the dark. (b) A $1.5 \mu \mathrm{m} \times 1.5 \mu \mathrm{m}$ map of the area marked by the gray box in (a). (c) Corresponding photovoltage buildup that links bright regions to dark regions in (b), indicating possible hole accumulation.

Figure S1. The measurements presented in this work were carried out on twin samples fabricated within the same batches as the high-efficiency devices. Figure la shows the scanning electron microscopy (SEM) image of the top surface of the mixed $\left(\mathrm{FAPbI}_{3}\right)_{0.85}\left(\mathrm{MAPbBr}_{3}\right)_{0.15}$ device consisting of crystal grains ranging from 100 to $500 \mathrm{~nm}$ in size. Figure $1 \mathrm{~b}-\mathrm{d}$ shows the elemental distributions of ${ }^{127} \mathrm{I},{ }^{79} \mathrm{Br}$, and ${ }^{12} \mathrm{C}$ across a $10 \mu \mathrm{m}$ $\times 10 \mu \mathrm{m}$ area of the perovskite surface obtained using the unique HIM-SIMS setup developed in 2015 by Wirtz et al. from the Luxembourg Institute of Science and Technology. ${ }^{14,15}$ The instrument allows high-sensitivity imaging of surfaces (surface sensitivity is $10-20 \mathrm{~nm}$ ) with lateral resolution down to $10 \mathrm{~nm}$, representing a factor of 5 enhancement with respect to the best commercially available SIMS instrument (Cameca NanoSIMS 50). Details can be found in the literature ${ }^{16}$ and the Supporting Information.

By mapping three isotopes, namely, ${ }^{127} \mathrm{I},{ }^{79} \mathrm{Br}$ and ${ }^{12} \mathrm{C}$, we observe that the ${ }^{79} \mathrm{Br}$ content varies from null/very low to high intensity, indicating compositional nonhomogeneity at the nanoscale. In the case of ${ }^{79} \mathrm{Br}$, several local minima extend to regions of up to $300 \mathrm{~nm}$ (Figure 1c). Local carbon hotspots are clearly revealed (Figure 1d), although a background ${ }^{12} \mathrm{C}$ signal due to vacuum contamination is also present. Combining the elemental maps of ${ }^{79} \mathrm{Br}$ and ${ }^{127} \mathrm{I}$ in Figure le reveals a clearer correlation. Most of the regions with null/very low ${ }^{79} \mathrm{Br}$ content correspond to regions with a high amount of ${ }^{127} \mathrm{I}$ ("blue spots"). A zoomed view of a region containing such a blue spot as well as the profiles of the ${ }^{79} \mathrm{Br}$ and ${ }^{127} \mathrm{I}$ signals along the dashed white lines are shown in Figure $1 \mathrm{f}-\mathrm{h}$. Although the SIMS technique is not quantitative, it clearly illustrates that ${ }^{127} \mathrm{I}$ counts totally prevail over ${ }^{79} \mathrm{Br}$ counts for at least $100 \mathrm{~nm}$. We exclude that such regions consist of pure $\mathrm{PbI}_{2}$ because they mostly correspond to the ${ }^{12} \mathrm{C}$ hotspots. This strongly underlines the presence of $\mathrm{FA} / \mathrm{MA}$ cations, ruling out the presence of $\mathrm{PbI}_{2}$. Since both MA and $\mathrm{FA}$ contain one $\mathrm{C}$ atom, the variation of the ${ }^{12} \mathrm{C}$ signal can be assigned to the difference of the corresponding SIMS signal obtained from $\mathrm{FA}_{x} \mathrm{MA}_{y} \mathrm{PbI}_{3}$ phase compared with the one that also contains ${ }^{79} \mathrm{Br}$. We suggest that the segregation of $\left(\mathrm{FAPbI}_{3}\right)_{0.85}\left(\mathrm{MAPbBr}_{3}\right)_{0.15}$ results in $a \mathrm{FA}_{x} \mathrm{MA}_{y} \mathrm{PbI}_{3}+b \mathrm{FA}_{x} \mathrm{MA}_{y} \mathrm{PbI}_{z} \mathrm{Br}_{3-z}+c \mathrm{FA}_{x} \mathrm{MA}_{y} \mathrm{PbBr}_{3}$, where $a, b$, and $c$ represent the relative weights of the phases. While $b$ dominates, HIM-SIMS shows that $a \neq 0$. $\mathrm{FA}_{x} \mathrm{MA}_{y} \mathrm{PbBr}_{3}$ is not revealed either because $c \approx 0$ or because those phases segregate below our resolution limit. In the first case, for mass conservation, the excess of $\mathrm{Br}$ can be contained in the $\mathrm{FA}_{x} \mathrm{MA}_{y} \mathrm{PbI}_{z} \mathrm{Br}_{3-z}$, where $z$ is slightly less than 0.85 . Overall, the analysis proves the nonhomogeneity in the elemental composition of the mixed perovskite surface and indicates that multiple interconnected perovskite phases with different halide contents are formed at nanometer scale. In order to determine whether the ${ }^{79} \mathrm{Br}$ and ${ }^{127} \mathrm{I}$ amounts vary through the depth of the capping layer, we also performed conventional SIMS depth profiling (without lateral resolution as in HIM-SIMS) of the capping layer (Figure S2). Interestingly, no significant gradient is observed. 
To confirm the surface nonhomogeneity and to investigate the influence of the composition on the charge carrier distribution, we carried out KPFM measurements. Figure 2 shows the results over the mixed cation/mixed halide perovskite surface (capping layer). A $5 \mu \mathrm{m} \times 5 \mu \mathrm{m} \mathrm{KPFM}$ surface potential map of the perovskite capping layer in the dark shows characteristics that can be immediately linked to the grain morphology (Figure S3a and Figure 1a). The map shows an average contact potential difference (CPD) of around 600 $\mathrm{mV}$. Interestingly, the map is not homogeneous but shows several darker spots with lower CPD (as indicated by the arrow in Figure 2a). A lower CPD value is related to a higher work function and therefore a deeper Fermi level. A $1.5 \mu \mathrm{m} \times 1.5 \mu \mathrm{m}$ map (Figure $2 b$ ) reveals that these lower-CPD regions overlap with well-defined crystal grains. In addition, darker stripes on the crystal grain facets also exhibit lower CPD values. In Figure $2 c$, corresponding to the difference of the map under illumination and in the dark, a clear trend is observed: high$\mathrm{CPD}$ regions in the dark lead to lower photovoltage, whereas low-CPD areas (e.g., the dark grain indicated by the arrow) are connected to higher photovoltage. These findings can be rationalized in the following way: the average $\mathrm{CPD}$ value of the mixed perovskite $(600 \mathrm{mV})$ is about $120 \mathrm{mV}$ higher than in the darker spots, where the CPD equals $480 \mathrm{mV}$. This value exactly matches the results for the pure $\mathrm{MAPbI}_{3}$ perovskite surface (see Figure S3f for comparison) and indicates the presence of nanodomains constituted of $\mathrm{FA}_{x} \mathrm{MA}_{y} \mathrm{PbI}_{3}$ perovskite phases on the surface of the mixed perovskite. In agreement, the higher photovoltage is caused by a slight downshift in the Fermi level under illumination, which could be due to hole accumulation within the I-rich perovskite grain. This correlates with the HIMSIMS measurements and demonstrates that the segregation of $\mathrm{FA}_{x} \mathrm{MA}_{y} \mathrm{PbI}_{3}$, extending up to about $300 \mathrm{~nm}$, is a fundamental property of the $\left(\mathrm{FAPbI}_{3}\right)_{0.85}\left(\mathrm{MAPbBr}_{3}\right)_{0.15}$ films (excluding the possibility of ion-beam-induced segregation). It is also worth noting that the chemical composition of the grain might vary with the crystal facet present at the surface, which may be responsible for the local change in CPD. However, this seems unlikely, as several flat crystals do not exhibit low CPD. Moreover, the possibility that the low-CPD grain (higher photovoltage) is composed of a precursor crystal (e.g., $\mathrm{PbI}_{2}$ ) is excluded by the unfavorable band alignment for hole accumulation. Overall, the measurements suggest compositional nonhomogeneities across the film at nanometer scale due to intrinsic halide segregation. These local nonhomogeneities are not usually revealed by standard characterization tools such as X-ray diffraction (XRD), Raman, and optical analysis, since they usually interrogate microscopic volumes of the sample. ${ }^{17}$ Indeed, no clear evidence for any phase segregation in the mixed perovskite layer could be observed by XRD (Figure S4). However, the relatively broad diffraction peaks could indicate the presence of domains in the perovskite having different phase compositions, as the change in the lattice parameters upon a small amount of $\mathrm{Br}$ substitution is expected to be small. On the other side, combined micro-Raman and microphotoluminescence (micro-PL) spectroscopy, although averaged over a diffraction-limited spot size of $300 \mathrm{~nm}$, can provide further information about local heterogeneities. ${ }^{18}$ The PL peak position map across the film over a $90 \mu \mathrm{m}^{2}$ area reveals an optical inhomogeneity (Figure 3a). Representative peakposition wavelengths at points 1,2 , and 3 are plotted in Figure $3 \mathrm{~b}$ along with those of the pristine $\mathrm{MAPbI}_{3}$ and $\mathrm{FAPbI}_{3}$ films. PL spectra and additional information are shown in Figures S5

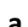

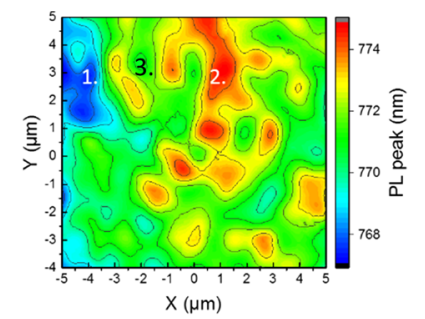

b
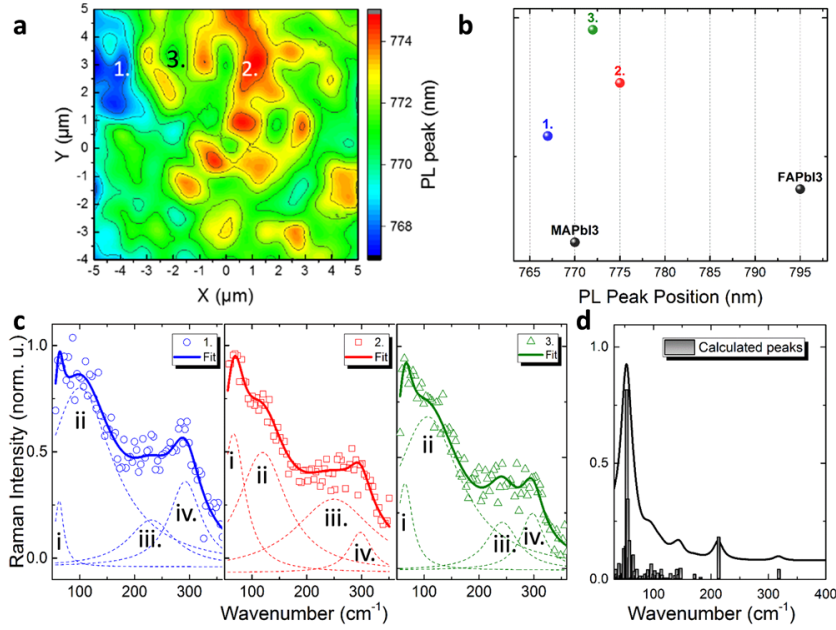

d
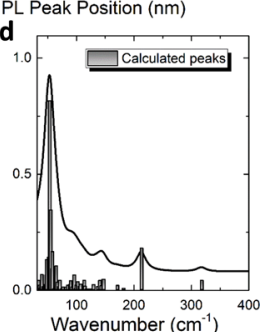

Figure 3. Optical and vibrational characterization. (a) Micro-PL peak shift map of the perovskite surface. Reabsorption effects are negligible because of the homogeneity in the thickness of the film. (b) PL peak positions of pure $\mathrm{MAPbI}_{3}$ and pure $\mathrm{FAPbI}_{3}$ compared with the selected points 1, 2, and 3 indicated in (a). (c) Micro-Raman spectra at points 1, 2, and 3. Solid lines are fits to the experimental data. The samples were encapsulated to prevent any air/moisture effects. (d) DFT-simulated Raman spectrum of the mixed perovskite.

and S6. Within the mixed cation/mixed halide (FAP$\left.\mathrm{bI}_{3}\right)_{0.85}\left(\mathrm{MAPbBr}_{3}\right)_{0.15}$ perovskite film, the peak positions of points 1-3 shift from 766 to $775 \mathrm{~nm}$. Although these measurements are averaged over an $\sim 300 \mathrm{~nm}$ spot, the shift can be related to a variation of the $\mathrm{Br}$ content with respect to I. ${ }^{19}$ In particular, point 2 exhibits a red-shifted emission similar to what is reported for $\mathrm{FA}_{y} \mathrm{MA}_{x} \mathrm{PbI}_{x} \mathrm{Br}_{3-x}$ with a minimal $\mathrm{Br}$ content. This further indicates the presence of halide redistribution within the film. On exactly the same area, we performed a structural investigation by means of micro-Raman spectroscopy. We collected the signals from points 1, 2, and 3 (Figure 3c) and in parallel performed density functional theory (DFT) analysis to support the mode assignments (Figure $3 \mathrm{~d}$ ). It should be noted that the Raman spectrum of the mixed perovskite film is here reported and simulated for the first time. The measured and DFT-calculated vibrational modes are listed in Table 1. Further information regarding the computational

Table 1. Fitted and DFT-Calculated Peaks (in $\mathrm{cm}^{-1}$ ) Associated with the Raman Spectra in Figure 3c

\begin{tabular}{cccccc} 
peak & 1 & 2 & 3 & calcd & \multicolumn{1}{c}{ mode assignment } \\
i & 62 & 66 & 65 & 50 & $\begin{array}{c}\mathrm{Pb}-\mathrm{I} / \mathrm{Pb}-\mathrm{Br} \text { bending and FA/MA } \\
\text { libration }\end{array}$ \\
ii & 100 & 119 & 105 & 100 & $\begin{array}{l}\mathrm{Pb}-\mathrm{I} / \mathrm{Pb}-\mathrm{Br} \text { stretching and FA/MA } \\
\text { libration }\end{array}$ \\
& & & & 150 & FA/MA libration \\
iii & 231 & 249 & 240 & 212 & FA torsion \\
iv & 292 & 297 & 297 & 317 & MA torsion \\
\hline
\end{tabular}

setup and geometries are presented in Experimental Methods and Figure S8. The Raman spectra of the three points consist of four main peaks (see Table 1). Similarly to the pristine $\mathrm{MAPbI}_{3}$ perovskite, $^{20,21}$ peaks $\mathrm{i}$ and ii represent combinations of the inorganic stretching and bending along with a minor contribution of the organic cations, while peaks iii and iv, a double feature not present in the $\mathrm{MAPbI}_{3}$ perovskite ${ }^{20,21}$ (see Figure S7), are related to a combination of FA and MA 
torsional modes linked together. We thus believe that this characteristic is unique to the mixed structure. The retrieved peak positions closely resemble the calculated Raman spectrum (Figure 3d). Comparing the three different points, we first observe that the relative amplitudes of peaks $\mathrm{i}$ and ii change. Peak ii dominates and is broader at point 1 , while its intensity and broadening are reduced at point 2 . An opposite trend is observed for peaks iii and iv, which are related to FA and MA vibrational modes, respectively. This variation can be induced by the structural inhomogeneity throughout the film. The slight shift in the mode frequencies can be due to the presence of different halides in the inorganic cage, which influence the organic motion through hydrogen bonds. ${ }^{21,22}$ Point 3 features characteristics intermediate between those of points 1 and 2 . Although this measurement does not aim to be exhaustive, it further supports the structural and compositional inhomogeneity across the film. Such inhomogeneity is responsible for the local PL variation, possibly induced by locally different perovskite structures or modified band gaps due to the different halide compositions.

Combining HIM-SIMS, KPFM, and micro-PL/Raman measurements, this study provides unprecedented insights into nanoscale properties of high-efficiency mixed cation/mixed halide perovskite solar cells. We have demonstrated that highly efficient perovskite devices are not constituted by a perfectly homogeneous crystalline structure, as a conventional thin-film solar cell would require, but that, contrary to expectations, nanoscale segregation of multiple perovskite compositions occurs. In particular, we have identified $\mathrm{FA}_{x} \mathrm{MA}_{y} \mathrm{PbI}_{3}$-rich domains within the mixed cation/mixed halide perovskite films extending up to hundreds of nanometers that can be more thermodynamically stable. We envisage that exploiting the concept of nanometer phase segregation, ultimately leading to a "bulk-heterojunction" perovskite solar cell, will be a potentially successful strategy for a significant advance in perovskite solar cell technology.

\section{ASSOCIATED CONTENT}

\section{S Supporting Information}

The Supporting Information is available free of charge on the ACS Publications website at DOI: 10.1021/jacs.6b10049.

Details of solar cell fabrication and device characteristics; SIMS depth profile; HIM-SIMS equipment details; KPFM, micro-Raman/PL, and XRD measurements; and computational details (PDF)

\section{AUTHOR INFORMATION}

\section{Corresponding Authors}

*giulia.grancini@epfl.ch

*mdkhaja.nazeeruddin@epfl.ch

ORCID

Giulia Grancini: 0000-0001-8704-4222

Jean-Nicolas Audinot: 0000-0002-4966-7653

Mohammad Khaja Nazeeruddin: 0000-0001-5955-4786

Notes

The authors declare no competing financial interest.

\section{ACKNOWLEDGMENTS}

I.Z. was supported by Qatar Environment and Energy Research Institute (QEERI), Hamad Bin Khalifa University (HBKU), and Qatar Foundation (Doha, Qatar). G.G. was supported by the cofunded Marie Skłodowska Curie Fellowship (H2020 Grant Agreement 665667, Fund 588072). The HIM-SIMS experiments performed at LIST were cofunded by the National Research Fund of Luxembourg (Grant C14/MS/8345352).

\section{REFERENCES}

(1) These are the top 10 emerging technologies of 2016. https:// www.weforum.org/agenda/2016/06/top-10-emerging-technologies2016 (accessed July 24, 2016).

(2) Li, X.; Bi, D.; Yi, C.; Décoppet, J.-D.; Luo, J.; Zakeeruddin, S. M.; Hagfeldt, A.; Grätzel, M. Science 2016, 353, 58.

(3) Jeon, N. J.; Noh, J. H.; Yang, W. S.; Kim, Y. C.; Ryu, S.; Seo, J.; Seok, S. I. Nature 2015, 517, 476.

(4) Jesper Jacobsson, T.; Correa-Baena, J.-P.; Pazoki, M.; Saliba, M.; Schenk, K.; Grätzel, M.; Hagfeldt, A. Energy Environ. Sci. 2016, 9, 1706.

(5) Pellet, N.; Gao, P.; Gregori, G.; Yang, T.-Y.; Nazeeruddin, M. K.; Maier, J.; Grätzel, M. Angew. Chem., Int. Ed. 2014, 53, 3151.

(6) Jeon, N. J.; Noh, J. H.; Kim, Y. C.; Yang, W. S.; Ryu, S.; Seok, S. I. Nat. Mater. 2014, 13, 897.

(7) Burschka, J.; Pellet, N.; Moon, S.-J.; Humphry-Baker, R; Gao, P.; Nazeeruddin, M. K.; Grätzel, M. Nature 2013, 499, 316.

(8) Roldán-Carmona, C.; Gratia, P.; Zimmermann, I.; Grancini, G.; Gao, P.; Graetzel, M.; Nazeeruddin, M. K. Energy Environ. Sci. 2015, 8, 3550.

(9) Saliba, M.; Matsui, T.; Seo, J.-Y.; Domanski, K.; Correa-Baena, J.P.; Nazeeruddin, M. K.; Zakeeruddin, S. M.; Tress, W.; Abate, A.; Hagfeldt, A.; Grätzel, M. Energy Environ. Sci. 2016, 9, 1989.

(10) Hoke, E. T.; Slotcavage, D. J.; Dohner, E. R.; Bowring, A. R.; Karunadasa, H. I.; McGehee, M. D. Chem. Sci. 2015, 6, 613.

(11) Yoon, S. J.; Draguta, S.; Manser, J. S.; Sharia, O.; Schneider, W. F.; Kuno, M.; Kamat, P. V. ACS Energy Lett. 2016, 1, 290.

(12) deQuilettes, D. W.; Zhang, W.; Burlakov, V. M.; Graham, D. J.; Leijtens, T.; Osherov, A.; Bulović, V.; Snaith, H. J.; Ginger, D. S.; Stranks, S. D. Nat. Commun. 2016, 7, 11683.

(13) Leijtens, T.; Hoke, E. T.; Grancini, G.; Slotcavage, D. J.; Eperon, G. E.; Ball, J. M.; De Bastiani, M.; Bowring, A. R.; Martino, N.; Wojciechowski, K.; McGehee, M. D.; Snaith, H. J.; Petrozza, A. Adv. Energy Mater. 2015, 5, 1500962.

(14) Wirtz, T.; Philipp, P.; Audinot, J.-N.; Dowsett, D.; Eswara, S. Nanotechnology 2015, 26, 434001.

(15) Wirtz, T.; Vanhove, N.; Pillatsch, L.; Dowsett, D.; Sijbrandij, S.; Notte, J. Appl. Phys. Lett. 2012, 101, 041601.

(16) Wirtz, T.; Dowsett, D.; Philipp, P. In Helium Ion Microscopy; Hlawacek, G., Gölzhäuser, A., Eds.; Springer: New York, 2016.

(17) Bi, D.; Tress, W.; Dar, M. I.; Gao, P.; Luo, J.; Renevier, C.; Schenk, K.; Abate, A.; Giordano, F.; Correa Baena, J.-P.; Decoppet, J.D.; Zakeeruddin, S. M.; Nazeeruddin, M. K.; Grätzel, M.; Hagfeldt, A. Sci. Adv. 2016, 2, e1501170.

(18) Draguta, S.; Thakur, S.; Morozov, Y. V.; Wang, Y.; Manser, J. S.; Kamat, P. V.; Kuno, M. J. Phys. Chem. Lett. 2016, 7, 715.

(19) Eperon, G. E.; Stranks, S. D.; Menelaou, C.; Johnston, M. B.; Herz, L. M.; Snaith, H. J. Energy Environ. Sci. 2014, 7, 982.

(20) Quarti, C.; Grancini, G.; Mosconi, E.; Bruno, P.; Ball, J. M.; Lee, M. M.; Snaith, H. J.; Petrozza, A.; Angelis, F. D. J. Phys. Chem. Lett. 2014, 5, 279.

(21) Grancini, G.; Marras, S.; Prato, M.; Giannini, C.; Quarti, C.; De Angelis, F.; De Bastiani, M.; Eperon, G. E.; Snaith, H. J.; Manna, L.; Petrozza, A. J. Phys. Chem. Lett. 2014, 5, 3836.

(22) Mosconi, E.; Amat, A.; Nazeeruddin, M. K.; Grätzel, M.; De Angelis, F. J. Phys. Chem. C 2013, 117, 13902. 